\title{
CORRIGENDUM
}

\section{Cytokine signals propagate through the brain}

L Vitkovic, JP Konsman, J Bockaert, R Dantzer, V Homburger and C Jacque. Mol Psychiatry 2000; 5: 604-615

The authors would like to correct the following phrase on page 607, second column, lines 6-7 from bottom:

'However, IL-1 $\beta$ concomitantly appears in the basolateral amygdala, where neuronal IL-1 receptors are present' should read:
'NF- $\kappa$ B concomitantly appears in the basolateral amygdala, where neuronal IL-1 receptors are present'.

The authors apologize for any inconvenience caused by this error. 